\title{
ON SEMI-REGULARIZATION TOPOLOGIES
}

\author{
M. MRŠEVIć, I. L. REILLY and M. K. VAMANAMURTHY
}

(Received 18 March 1983)

Communicated by J. M. Rubinstein

\begin{abstract}
This paper discusses several properties of topological spaces and how they are reflected by corresponding properties of the associated semi-regularization topologies. For example a space is almost locally connected if and only if its semi-regularization is locally connected. Various separation, connectedness, covering, and mapping properties are considered.

1980 Mathematics subject classification (Amer. Math. Soc.): 54 A 10, 54 C 10, 54 D 05, 54 D 10, 54 D 30.

Keywords and phrases: semi-regular, regular open, almost regular, almost locally connected, nearly compact, almost continuous, $\delta$-continuous, super-continuous.
\end{abstract}

\section{Introduction}

In this paper we are concerned with the relationship between the properties of a topological space $(X, \mathscr{T})$ and those of its semi-regularization topology $\left(X, \mathscr{T}_{s}\right)$. Cameron [2] has called a topological property $R$ semi-regular provided that $(X, \mathscr{T})$ has property $R$ if and only if $\left(X, \mathscr{T}_{s}\right)$ has property $R$. In one sense this paper is a continuation of the study of semi-regular properties.

Porter and Thomas [20] and Velicko [29] have shown the importance of semi-regularization topologies in the study of $H$-closed and minimal Hausdorff spaces. Cameron [2] has applied semi-regularization techniques to the study of $S$-closed spaces. Here we try to give a systematic discussion of several topological

The first author acknowledges the support of a University of Auckland Post-Doctoral Fellowship, and the second author acknowledges a grant from the University of Auckland Research Fund.

(c) 1985 Australian Mathematical Society 0263-6115/85 \$A2.00+0.00 
properties which have been introduced in the past few years by means of studying the associated semi-regularization topologies.

In Section 2 we give a brief outline of the relevant basic properties of semi-regularization topologies. Section 3 discusses separation properties, and shows that the relationship between $(X, \mathscr{T})$ and $\left(X, \mathscr{T}_{s}\right)$ undergoes an interesting change as we progress along the hierarchy of separation properties. In particular, the condition that $\left(X, \mathscr{T}_{s}\right)$ be $T_{1}$ (or $\left.T_{0}\right)$ is stronger than requiring $(X, \mathscr{T})$ to be $T_{1}$ (or $\left.T_{0}\right)$; for Hausdorff $\left(T_{2}\right)$ and Urysohn $\left(T_{2 \frac{1}{2}}\right)$ these conditions are equivalent, while the condition $\left(X, \mathscr{T}_{s}\right)$ being (completely) regular is weaker than requiring $(X, \mathscr{T})$ to be (completely) regular. In Section 4 we are concerned with connectedness properties, and some of our results improve some recent theorems of Macuso [12]. Section 5 is a short collection of results dealing with covering properties which have appeared scattered in the literature, but which fit nicely into our framework. The material in Section 6 indicates how some recent work on variations of continuity can be interpreted in this setting by suitable changes of topology on either domain or codomain spaces. We provide a small sample of the possible results which can be generated by taking this approach.

The topological spaces are not assumed to satisfy any separation axioms, except those explicitly stated.

\section{Semi-regular topologies}

In a topological space $(X, \mathscr{T})$ a set $A$ is called $\mathscr{T}$ regular open if $A=$ $\mathscr{T} \operatorname{int}(\mathscr{T} \mathrm{cl} A)$ and $\mathscr{T}$ regular closed if $A=\mathscr{T} \operatorname{cl}(\mathscr{T}$ int $A)$. We let $R O(X, \mathscr{T})$ denote the collection of all regular open sets in $(X, \mathscr{T})$. Since the intersection of two regular open sets is regular open, the family of $\mathscr{T}$ regular open sets forms a base for a smaller topology $\mathscr{T}_{s}$ on $X$, called the semi-regularization of $\mathscr{T}$. The space $(X, \mathscr{T})$ is said to be semi-regular if $\mathscr{T}_{s}=\mathscr{T}$. Any regular space is semi-regular, but the converse is false.

In [29], Velicko introduced the notion of $\delta$-open and $\delta$-closed sets in a space $(X, \mathscr{T})$. A point $x \in X$ is said to be a $\delta$-cluster point of the subset $A$ of $(X, \mathscr{T})$ if $A \cap U \neq \varnothing$ for every $\mathscr{T}$ regular open set $U$ containing $x$. The set of all $\delta$-cluster points of $A$ is called the $\delta$-closure of $A$ and denoted by $[A]_{\delta}$. If $A=[A]_{\delta}$ then $A$ is called $\delta$-closed, and the complement of a $\delta$-closed set is called $\delta$-open. For any space $(X, \mathscr{T})$ the collection of all $\delta$-open sets forms a topology $\mathscr{T}_{\delta}$ on $X$, and the definitions of $\delta$-adherent point, $\delta$-closure, $\delta$-convergence of filterbases and so on are the usual definitions applied to the topology $\mathscr{T}_{\delta}$. A basic fact which we shall exploit is that $\mathscr{T}_{\delta}=\mathscr{T}_{s}$. 
Throughout this paper we use the Bourbaki notation $\mathscr{T}_{\alpha A}$ to denote $\mathscr{T} \operatorname{int}(\mathscr{T} \mathrm{cl} A)$, often suppressing the $\mathscr{T}$ when there is no confusion possible. We now give some basic results for semi-regularization topologies that we shall require. It is clear that any property which is preserved by enlargement (or expansion) of topologies will be carried over from $\left(X, \mathscr{T}_{s}\right)$ to $(X, \mathscr{T})$.

LEMMA 1. Bourbaki [1, 3 a) and d), page 117]. If $A$ and $B$ are disjoint open sets in $(X, \mathscr{T})$, then $\mathscr{T} \alpha(A)$ and $\mathscr{T} \alpha(B)$ are disjoint open sets in $\left(X, \mathscr{T}_{s}\right)$ containing $A$ and $B$ respectively.

Lemma 2. Katětov [7, Satz 1.3]. Let $(Y, \mathscr{U})$ be a regular space. If $f:(X, \mathscr{T}) \rightarrow$ $(Y, \mathscr{U})$ is continuous, then $f:\left(X, \mathscr{T}_{s}\right) \rightarrow(Y, \mathscr{U})$ is continuous.

Lemma 3. Herrington [5, Remark 2.1]. Semi-regularization topologies are preserved by topological (Tychonoff) products.

While semi-regularization topologies behave well with respect to products, they do not behave well with respect to subspaces. For example, we can have the extreme case of $A \subset(X, \mathscr{T})$ and $\mathscr{T}_{s} / A$ being indiscrete while $(\mathscr{T} / A)_{s}$ is discrete. Choose $(X, \mathscr{T})$ to be an infinite set with the cofinite topology and $A$ to be any finite subset of $X$. Our next result shows that for two classes of subsets all is well.

LEMMA 4. Let $A \subset(X, \mathscr{T})$. If $A$ is open or dense then

(a) $R O(A, \mathscr{T} / A)=\{V \cap A: V \in R O(X, \mathscr{T})\}$

(b) $(\mathscr{T} / A)_{s}=\mathscr{T}_{s} / A$.

Proof. We need only prove (a), since (b) follows immediately from (a).

First let $A$ be open and let $W \in R O(A, \mathscr{T} / A)$. Then $(\mathscr{T} / A) \operatorname{cl}(W)=\mathscr{T} \mathrm{cl}(W) \cap$ $A$, hence $(\mathscr{T} / A) \alpha(W)=(\mathscr{T} \alpha(W)) \cap A=V \cap A$, where $V=\mathscr{T} \alpha(W) \in$ $R O(X, \mathscr{T})$

Conversely, let $V \in R O(X, \mathscr{T})$ and $W=V \cap A$. Then $(\mathscr{T} / A) \alpha(W)=$ $\mathscr{T} \operatorname{int}(\mathscr{T} \operatorname{cl}(V \cap A) \cap A)=\mathscr{T} \operatorname{int}(\mathscr{T} \operatorname{cl}(V) \cap A)=\mathscr{T} \alpha(V) \cap A=V \cap A=W$, hence $W \in R O(A, \mathscr{T} / A)$.

Next let $A$ be dense. Then the result follows from the proof of Theorem 4.1 in [22].

The last result we require is the fact that the process of semi-regularizing a topological space is an idempotent operation. The proof of this depends on the observation that the family of all regularly open subsets of $\left(X, \mathscr{T}_{s}\right)$ coincides with the collection of all regularly open subsets of $(X, \mathscr{T}),([1$, page $138,20 \mathrm{~b}]$ and $[20$, 12.13]).

LEMma 5. For any topological space $(X, \mathscr{T}),\left(\mathscr{T}_{s}\right)_{s}=\mathscr{T}_{s}$. 


\section{Separation properties}

The fact that the Hausdorff property is shared by a topological space and its semi-regularization has been known for some time, for example see Bourbaki [1, page 138]. A proof of Proposition 1 comes immediately from Lemma 1 and the general comment about properties preserved by enlargements of topologies.

Proposition 1. $(X, \mathscr{T})$ is Hausdorff if and only if $\left(X, \mathscr{T}_{s}\right)$ is Hausdorff.

The situation is quite different for the $T_{0}$ and $T_{1}$ properties.

Proposition 2. If $\left(X, \mathscr{T}_{s}\right)$ is $T_{0}$ (respectively $\left.T_{1}\right)$ then $(X, \mathscr{T})$ is $T_{0}$ (respectively $\left.T_{1}\right)$, but the converse is false.

Proof. The positive statement follows from the containment $\mathscr{T}_{s} \subset \mathscr{T}$. Next, let $(X, \mathscr{T})$ be an infinite set with the cofinite topology. Then $(X, \mathscr{T})$ is $T_{1}$, but $\left(X, \mathscr{T}_{s}\right)$ is the indiscrete topology and so is not even $T_{0}$.

On the other hand, the Urysohn (or $T_{2 \frac{1}{2}}$ ) property behaves like $T_{2}$. Steen and Seeback [27, pages 13 and 15] call a Urysohn space by the term completely Hausdorff, and use Urysohn for spaces called functionally Hausdorff by other authors.

Proposition 3 [7, Remark 3.4]. ( $X, \mathscr{T})$ is Urysohn (respectively, functionally Hausdorff) if and only if $\left(X, \mathscr{T}_{s}\right)$ is Urysohn (respectively functionally Hausdorff).

Proof. One implication is clear since $T_{2 \frac{1}{2}}$ is preserved by enlargement [27, page 14]. Conversely, let $(X, \mathscr{T})$ be Urysohn and $a, b$ be distinct points of $X$. There are $\mathscr{T}$ open sets $U$ and $V$ such that $a \in U, b \in V$ and $\bar{U} \cap \bar{V}=\varnothing$. Then $\alpha(U)$ and $\alpha(V)$ are $\mathscr{T}_{s}$ open, $a \in U \subset \alpha(U)$ and $b \in V \subset \alpha(V)$, and

$$
\mathscr{T}_{s} \operatorname{cl}(\alpha(U)) \cap \mathscr{T}_{s} \operatorname{cl}(\alpha(V))=\mathscr{T} \operatorname{cl}(\alpha(U)) \cap \mathscr{T} \operatorname{cl}(\alpha(V)) \subset \bar{U} \cap \bar{V}=\varnothing .
$$

Thus $\left(X, \mathscr{T}_{s}\right)$ is Urysohn. The proof for functionally Hausdorff follows from Lemma 2.

As Steen and Seeback have observed [27, Example 66] the higher separation properties are not in general preserved by enlargement of topologies. This is one reason for the very different relationship between the (complete) regularity of $(X, \mathscr{T})$ and $\left(X, \mathscr{T}_{s}\right)$, from that exhibited by the Hausdorff property in Proposition 1 . 
The class of almost regular spaces was introduced and studied by Singal and Arya [22].

DEFINITION 1. $(X, \mathscr{T})$ is said to be almost regular if for each $\mathscr{T}$ regularly closed subset $A$ of $X$ and each point $x \in X-A$ there are disjoint $\mathscr{T}$ open sets $U$ and $V$ such that $A \subset U$ and $x \in V$.

We observe that Singal and Arya [22, Example 3.1 and Remark 3.2] have shown that there are almost regular spaces which are not regular, and that almost regularity and semi-regularity are independent notions. Our next result provides the fundamental relationship between these concepts, and was noted by Hermann [9, page 218].

THEOREM 1. $(X, \mathscr{T})$ is almost regular if and only if $\left(X, \mathscr{T}_{s}\right)$ is regular.

Proof. Let $(X, \mathscr{T})$ be almost regular, $C$ be a $\mathscr{T}_{s}$ closed subset of $X$ and $x \in X-C$. Now $C=\cap\left\{C_{i}: i \in I\right\}$ where $C_{i}$ is $\mathscr{T}$ regularly closed for each $i \in I$. Thus there is some $j \in I$ such that $x \in X-C_{j}$. So there are disjoint $\mathscr{T}$ open sets $U$ and $V$ such that $C \subset C_{j} \subset U$ and $x \in V$. By Lemma 1 , there are disjoint $\mathscr{T}_{s}$ open sets $U^{\prime}$ and $V^{\prime}$ such that $C \subset U \subset U^{\prime}$ and $x \in U \subset U^{\prime}$. Hence $\left(X, \mathscr{T}_{s}\right)$ is regular.

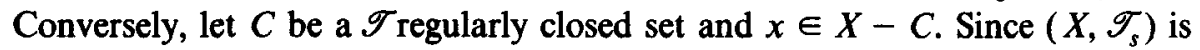
regular, there are disjoint $\mathscr{T}_{s}$ open sets $U$ and $V$ such that $C \subset U$ and $x \in V$. Since $\mathscr{T}_{s} \subset \mathscr{T},(X, \mathscr{T})$ is almost regular.

COROLlaRY 1. Regularity is not a semi-regular property, but almost regularity is.

Proof. The half-disc topology [27, Example 78] is a space $(X, \mathscr{T})$ which is not regular, while its semi-regularization $\left(X, \mathscr{T}_{s}\right)$ is the usual topology on the closed upper half plane, and so $\left(X, \mathscr{T}_{s}\right)$ is regular, indeed metrizable.

Applying Theorem 1 to the space $\left(X, \mathscr{T}_{s}\right)$ and using Lemma 5 shows that $\left(X, \mathscr{T}_{s}\right)$ is almost regular if and only if it is regular. But from Theorem $1,\left(X, \mathscr{T}_{s}\right)$ is regular if and only if $(X, \mathscr{T})$ is almost regular. Thus almost regularity is a semi-regular property.

COROLlaRY 2 [22, Theorem 3.1]. $(X, \mathscr{T})$ is semi-regular and almost regular if and only if it is regular.

Papic [19, Theorem 2] has proved one half of Theorem 1 for Hausdorff spaces. Theorem 1 can be used to give direct alternative proofs of several of the results of Singal and Arya [22]. For example, the characterizations of almost regularity 
given in [22, Theorem 2.2] can be obtained from Theorem 1 and standard characterizations of regularity. Indeed, the result can be generalized by replacing regularly open (closed) by $\mathscr{T}_{s}$ open (closed) throughout. Similarly, their theorem for products of almost regular spaces [22, Theorem 5.1] follows directly from Theorem 1 above, the fact that regularity is preserved by products, and Lemma 3. Lemma 4 and the fact that regularity is hereditary shows that almost regularity is inherited by open subspaces and dense subspaces (see [22, Theorems 4.1 and 4.2]). Papic [19, Theorem 4] has proved the result for open subspaces by quite a different method.

We can use our previous results to obtain an improvement of [22, Theorem 3.2]. Propositions 1 and 2 indicate that $(X, \mathscr{T})$ is Hausdorff is a stronger condition than $\left(X, \mathscr{T}_{s}\right)$ is $T_{0}$.

Proposition 4. If $(X, \mathscr{T})$ is almost regular and $\left(X, \mathscr{T}_{s}\right)$ is $T_{0}$ then $(X, \mathscr{T})$ is Urysohn.

Proof. We have that $\left(X, \mathscr{T}_{s}\right)$ is regular by Theorem 1 , and $T_{0}$, and therefore it is $T_{3}$ and hence $T_{2 \frac{1}{2}}$. So Proposition 3 implies that $(X, \mathscr{T})$ is $T_{2 \frac{1}{2}}$.

Almost completely regular spaces were introduced by Singal and Arya [23], and studied there and in [25].

Definition 2. $(X, \mathscr{T})$ is almost completely regular if for each $\mathscr{T}$ regularly closed set $A$ and for each point $x \in X-A$ there is a continuous function $f:(X, \mathscr{T}) \rightarrow$ $[0,1]$ such that $f(x)=1$ and $f(A)=0$.

The relationship between $(X, \mathscr{T})$ and $\left(X, \mathscr{T}_{s}\right)$ is this context has been considered by Singal and Mathur [25]. Our next result improves their Theorem 2.1.

THEOREM 2. $(X, \mathscr{T})$ is almost completely regular if and only if $\left(X, \mathscr{T}_{s}\right)$ is completely regular.

Proof. Let $K$ be a $\mathscr{T}_{s}$ closed set and $x \in X-K$. So $K=\bigcap\left\{A_{\alpha}: \alpha \in \Delta\right\}$ where each $A_{\alpha}$ is $\mathscr{T}$ regularly closed, and $x \in X-A_{\beta}$ for some $\beta \in \Delta$. Since $(X, \mathscr{T})$ is almost completely regular, there is a continuous function $f:(X, \mathscr{T}) \rightarrow[0,1]$ such that $f(x)=1$ and $f\left(A_{\beta}\right)=0$. Now $[0,1]$ is regular, so that by Lemma $2, f$ : $\left(X, \mathscr{T}_{s}\right) \rightarrow[0,1]$ is continuous. Furthermore, $f(x)=1$ and $K \subset A_{\beta}$ implies $f(K)$ $=0$. Hence $\left(X, \mathscr{T}_{s}\right)$ is completely regular.

The converse is [25, Corollary 2.1]. 
Again, we can use Theorem 2 to provide direct alternative proofs of several of the results of [23] and [25]. For example, [25, Theorem 2.2] which is a generalization of Theorem 3.5 of [23], follows from Theorem 2, Lemma 2 and standard characterizations of complete regularity. The theorem for products of almost completely regular spaces [23, Theorem 3.9] follows from Theorem 2, Lemma 3 and the fact that complete regularity is preserved by products. Since complete regularity is hereditary, Lemma 4 shows that any open subspace of an almost completely regular spaces is almost completely regular. An alternative proof has been given for this result by Singal and Mathur [25, Theorem 3.1].

Using Theorem 2 and Lemma 5 we can see that although complete regularity is not a semi-regular property, almost complete regularity is.

\section{Connectedness properties}

The fact that a space and its semi-regularization topology share connectedness follows from the next result. We denote by $\operatorname{Clo}(X, \mathscr{T})$ the collection of all subsets of $(X, \mathscr{T})$ which are both $\mathscr{T}$ open and $\mathscr{T}$ closed.

Proposition 5. $\operatorname{Clo}(X, \mathscr{T})=\operatorname{Clo}\left(X, \mathscr{T}_{s}\right)$.

Proof. If $G \in \operatorname{Clo}(X, \mathscr{T})$ then $G$ is $\mathscr{T}$ regularly open and hence $\mathscr{T}_{s}$ open. Furthermore $X-G$ is also $\mathscr{T}_{s}$ open. Thus $G \in \operatorname{Clo}\left(X, \mathscr{T}_{s}\right)$. On the other hand, $\operatorname{Clo}\left(X, \mathscr{T}_{s}\right) \subset \operatorname{Clo}(X, \mathscr{T})$ since $\mathscr{T}_{s} \subset \mathscr{T}$.

COROLlaRY 3. $(X, \mathscr{T})$ is connected if and only if $\left(X, \mathscr{T}_{s}\right)$ is connected.

While connectedness is a semi-regular property, path connectedness is not.

Proposition 6. If $(X, \mathscr{T})$ is path connected then $\left(X, \mathscr{T}_{s}\right)$ is path connected, but the converse is false.

Proof. Since $\mathscr{T}_{s} \subset \mathscr{T}$, if $f:[0,1] \rightarrow X$ is a path in $(X, \mathscr{T})$ then $f$ is a path in $\left(X, \mathscr{T}_{s}\right)$. However, if $(X, \mathscr{T})$ is a countably infinite set with the cofinite topology then it is not path connected [27, Example 18]. But $\left(X, \mathscr{T}_{s}\right)$ is indiscrete and hence is path connected. A more complex example is the countable complement extension topology $(X, \mathscr{T})$ on the real line described by Steen and Seebach [27, Example 63]. Then $(X, \mathscr{T})$ is totally pathwise disconnected, while $\left(X, \mathscr{T}_{s}\right)$ is the usual topology on the real line and so is path connected.

Proposition 7. Let $A \subset(X, \mathscr{T})$. If $A$ is $\mathscr{T}_{\text {s }}$ disconnected then $A$ is $\mathscr{T}$ disconnected, and the converse is true if $A$ is $\mathscr{T}$ open, but false in general. 
Proof. That $A$ is $\mathscr{T}_{s}$ disconnected implies $A$ is $\mathscr{T}$ disconnected follows directly from $\mathscr{T}_{s} \subset \mathscr{T}$.

If $A$ is $\mathscr{T}$ open and $\mathscr{T}$ disconnected then $A=G \cup H$ where $G$ and $H$ are disjoint $\mathscr{T}$ open non-empty sets. Since $A$ is $\mathscr{T}$ open, $A \subset \alpha(G) \cup \alpha(H)$, and $\alpha(G)$ and $\alpha(H)$ are disjoint $\mathscr{T}_{s}$ open non-empty sets, so that $A$ is $\mathscr{T}_{s}$ disconnected.

If $(X, \mathscr{T})$ is an infinite set with the cofinite topology then $\mathscr{T}_{s}$ is the indiscrete topology. Let $a \neq b \in X$ and $A=\{a, b\}$. Then $A$ is $\mathscr{T}$ disconnected but $A$ is $\mathscr{T}_{s}$ connected.

DEFINITION 3. $(X, \mathscr{T})$ is called weakly locally connected if each component of $(X, \mathscr{T})$ is $\mathscr{T}$ open ([1], page 156 and [14], Section 3).

We observe that every connected space is weakly locally connected, and every locally connected space is weakly locally connected, but the converses are false. The next result follows immediately from Theorem 5 of [14] and Proposition 7 above.

Proposition 8. $(X, \mathscr{T})$ is weakly locally connected if and only if $\left(X, \mathscr{T}_{s}\right)$ is weakly locally connected, that is, weakly locally connected is a semi-regular property.

We note that Cameron $[2$, Theorem 10$]$ has proved that $(X, \mathscr{T})$ is extremally disconnected if and only if $\left(X, \mathscr{T}_{s}\right)$ is extremally disconnected.

The class of almost locally connected spaces has been recently introduced and studied by Mancuso [12].

DefinITION 4. $(X, \mathscr{T})$ is almost locally connected at a point $p \in X$ if each $\mathscr{T}$ regularly open neighbourhood of $p$ contains a connected $\mathscr{T}$ open neighbourhood of $p .(X, \mathscr{T})$ is almost locally connected if it is almost locally connected at each of its points.

Mancuso [12, Example 3.4] has cited the countable complement extension topology on the reals [27, Example 63] to show that there are spaces which are almost locally connected and not locally connected. Our next result gives the relationship between these concepts.

THEOREM $3(X, \mathscr{T})$ is almost locally connected if and only if $\left(X, \mathscr{T}_{s}\right)$ is locally connected.

Proof. Let $(X, \mathscr{T})$ be almost locally connected, $p \in X$ and $G$ be a $\mathscr{T}_{s}$ open neighbourhood of $p$. There is a $\mathscr{T}$ regularly open neighbourhood $V$ of $p$ such that $V \subset G$. By Definition 4 there is a $\mathscr{T}$ connected $\mathscr{T}$ open set $W$ such that $p \in W \subset V$. 
Thus $p \in W \subset \mathscr{T} \alpha(w) \subset \mathscr{T} \alpha(V)=V \subset G$, and $\mathscr{T} \alpha(W)$ is $\mathscr{T}$ connected. Hence $\mathscr{T} \alpha(W)$ is a $\mathscr{T}_{s}$ connected and $\mathscr{T}_{s}$ open neighbourhood of $p$, so that $\left(X, \mathscr{T}_{s}\right)$ is locally connected.

Conversely, let $p \in X$ and $G$ be a $\mathscr{T}$ regularly open neighbourhood of $p$. Since $\left(X, \mathscr{T}_{s}\right)$ is locally connected there is a $\mathscr{T}_{s}$ open $\mathscr{T}_{s}$ connected neighbourhood $W \subset G$ of $p$. By Proposition $7, W$ is $\mathscr{T}$ connected, so that $(X, \mathscr{T})$ is almost locally connected at $p$.

Corollary 4. If $(X, \mathscr{T})$ is locally connected then $\left(X, \mathscr{T}_{s}\right)$ is locally connected, but the converse is false.

Corollary 5 [12, Proposition 3.3]. If $(X, \mathscr{T})$ is semi-regular then it is locally connected if and only if it is almost locally connected.

Theorem 3 can be used to provide direct alternative proofs of some of the results of Mancuso [12]. For example, his product theorem for almost locally connected spaces [12, Theorem 3.21] follows from Theorem 3, Corollary 3, the corresponding result for local connectedness [5, 4.3, page 113] and Lemma 3. Theorem 3 also allows us to improve some of the theorems of [12]. The next result generalizes Theorem 3.5 of [12], and is proved by appealing to Theorem 3, Proposition 7, and the corresponding result for local connectedness [5, Theorem 4.2 page 113].

PROPOSITION 9. $(X, \mathscr{T})$ is almost locally connected if and only if components of $\mathscr{T}_{s}$ open sets are $\mathscr{T}_{s}$ open.

Remark 3.6(b) of Mancuso [12] indicates that any almost locally connected space is weakly locally connected, and this is also immediate from the previous proposition. We now provide an example of a space which is weakly locally connected but not almost locally connected.

EXAmple. Let $(X, \mathscr{T})$ be the space $\mathbf{Q} \cup\{\sqrt{2}\}$ where $\mathbf{Q}$, the rationals, has the usual topology as a subspace of $\mathbf{R}$, while each open neighbourhood of $\sqrt{2}$ is a cofinite set. Then $(X, \mathscr{T})$ is $T_{1}$, connected and hence weakly locally connected. However $X-\{\sqrt{2}\}=\mathbf{Q}$ is totally disconnected, thus is not weakly locally connected, and therefore is not almost locally connected. Hence by Mancuso [12, Theorem 3.9], $(X, \mathscr{T})$ is not almost locally connected.

The above example shows that the weakly locally connected analogue of $[12$, Theorem 3.9] is false, and provides a countable space $(X, \mathscr{T})$ which is a $T_{1}$, 
weakly locally connected, compact, nondiscrete space with a dispersion point. Compare [12, Corollary 3.10].

Lemma 4 and Theorem 3 can be used to prove the following improvement of [12, Theorem 3.8].

Proposition 10. Let A be a $\mathscr{T}_{s}$ open subspace of an almost locally connected space $(X, \mathscr{T})$. Then $(A, \mathscr{T} / A)$ is almost locally connected.

Theorem 3 and Lemma 5 reveal that almost locally connected is a semi-regular property.

\section{Covering properties}

The class of nearly compact spaces was introduced by Singal and Mathur [24], and has since been considered by several authors, see $[3,6,10,15,28$ for example]. A space $(X, \mathscr{T})$ is called nearly compact if every open cover of $X$ has a finite subfamily, the interiors of the closures of whose members cover $X$. The fundamental relationship between this concept and the compactness of $\left(X, \mathscr{T}_{s}\right)$ was given by Carnahan [3, Theorem 4.1].

THEOREM 4. $(X, \mathscr{T})$ is nearly compact if and only if $(X, \mathscr{T})$ is compact.

A space $(X, \mathscr{T})$ is called nearly paracompact [21] if every $\mathscr{T}$ regularly open cover of $X$ has a locally finite open refinement. If $\left(X, \mathscr{T}_{s}\right)$ is paracompact it is clear that $(X, \mathscr{T})$ is nearly paracompact. The converse can be proved by using the following characterization given by Singal and Arya [21]. A space $(X, \mathscr{T})$ is nearly paracompact if and only if every $\mathscr{T}$ regularly open cover of $X$ has a locally finite $\mathscr{T}$ regularly open refinement. So we have the following result which was proved by another method by Noiri [17, Theorem 3].

THEOREM 5. $(X, \mathscr{T})$ is nearly paracompact if and only if $\left(X, \mathscr{T}_{s}\right)$ is paracompact.

The existence of spaces which are nearly compact but not compact [24], and nearly paracompact but not paracompact [21, Example 1.1], show that compactness and paracompactness are not semi-regular properties.

The notion of nearly compactness was localized by Carnahan [3], by introducing $N$-closed subsets of a topological space. The subset $A$ of $(X, \mathscr{T})$ is said to be $N$-closed if for every cover $\mathscr{U}$ of $A$ by $\mathscr{T}$ open sets in $X$, there is a finite subfamily $\left\{U_{1}, U_{2}, \ldots, U_{n}\right\}$ of $\mathscr{U}$ such that $A \subset \cup\left\{\mathscr{T}_{\alpha} U_{i}: i=1,2, \ldots, n\right\}$. Noiri [15, Theorem 
3:1] showed that $A$ is $N$-closed in $(X, \mathscr{T})$ if and only if $A$ is compact in $\left(X, \mathscr{T}_{s}\right)$. Thus $(X, \mathscr{T})$ can be defined to be locally nearly compact if each point of $X$ has an open neighbourhood whose closure is a nearly compact subset of $(X, \mathscr{T})$. The proof of Lemma 2.1 of Herrington [6] reveals that if $\left(X, \mathscr{T}_{s}\right)$ is locally compact then $(X, \mathscr{T})$ is locally nearly compact. The converse has been proved by Carnahan [3, Theorem 4.5] with the additional condition that $(X, \mathscr{T})$ is Hausdorff. The next result generalizes this to arbitrary topological spaces.

THEOREM 6. $(X, \mathscr{T})$ is locally nearly compact if and only if $\left(X, \mathscr{T}_{s}\right)$ is locally compact.

Proof. Let $(X, \mathscr{T})$ be locally nearly compact and let $x \in X$. Then there is an open neighbourhood $U$ of $x$ such that $\mathscr{T} \mathrm{cl} U$ is nearly compact in $(X, \mathscr{T})$. The regularly open set $V=\mathscr{T} \alpha U$ is a $\mathscr{T}_{s}$ open neighbourhood of $x$ and $\mathscr{T}_{s} \mathrm{cl} V=\mathscr{T} \mathrm{cl} V$ $=\mathscr{T} \mathrm{cl} U$. Hence $\mathscr{T}_{s} \mathrm{cl} V$ is compact in $\left(X, \mathscr{T}_{s}\right)$ by [10, Theorem 3.1]. Thus $\left(X, \mathscr{T}_{s}\right)$ is locally compact.

The converse statement is proved in [6, Lemma 2.1].

These theorems can provide direct proofs of many of the results in the literature but only Herrington [6, Theorems 2.1,3.5] seems to have taken this approach. For example, most of the characterizations of nearly compact spaces given by Joseph [10] and Thompson [28] follow from Theorem 4, standard results for compact spaces and the observation that $\alpha$-convergence [10] and $\omega$-convergence [28] of a filterbase $\mathscr{F}$ in $(X, \mathscr{T})$ are equivalent to convergence of $\mathscr{F}$ in $\left(X, \mathscr{T}_{s}\right)$. The well-known result that the product of a compact space and a paracompact space is paracompact, Lemma 3 and Theorems 4 and 5, give a direct proof of Theorem 5.1 of Singal and Arya [21]. The next result improves Theorem 4.1 of Noiri [15] and Theorem 4.1 of Singal and Mathur [25].

Proposition 11. Every locally nearly compact Hausdorff space is almost completely regular.

Proof. By Theorem 6 and Proposition $1,\left(X, \mathscr{T}_{s}\right)$ is locally compact Hausdorff, and therefore completely regular $[5,6.4$, page 238$]$. Hence by Theorem $2,(X, \mathscr{T})$ is almost completely regular.

Cameron [2] has shown that there are some covering properties which are semi-regular properties, namely quasi- $H$-closed and $S$-closed. $(X, \mathscr{T})$ is called quasi- $H$-closed ( $S$-closed) if every open (semiopen) cover of $X$ has a finite subfamily whose closures cover $X$. 
We can use Theorems 4 and 5 together with Lemma 5 to show that nearly compactness and nearly paracompactness are also semi-regular properties.

\section{Mapping properties}

Three variations of continuity which have been studied recently are relevant to our considerations. Almost continuity was introduced by Singal and Singal [26], $\delta$-continuity by Noiri [18] and super-continuity by Munshi and Bassan [13], and the basic properties of these classes of mappings were investigated in these papers.

Definition 5. A function $f:(X, \mathscr{T}) \rightarrow(Y, \mathscr{U})$ is called

i) almost continuous (abbreviated as $\mathrm{AC}$ )

ii) $\delta$-continuous

iii) super-continuous (abbreviated as SC)

if for each $x \in X$ and each $\mathscr{U}$ open set $V$ containing $f(x)$ there is a $\mathscr{T}$ open set $U$ containing $x$ such that

i) $f(U) \subset \mathscr{U} \alpha V$,

ii) $f(\alpha U) \subset \mathscr{Q} \alpha V$,

iii) $f(\alpha U) \subset V$, respectively.

Provided the domain or codomain spaces (or both) are retopologized appropriately, then these three concepts coincide with the usual notion of continuity. Specifically, we have the following result whose proof is straightforward.

Proposition 12. Let $f:(X, \mathscr{T}) \rightarrow(Y, \mathscr{U})$ be a function. Then

i) $f$ is $A C$ if and only if $f:(X, \mathscr{T}) \rightarrow\left(Y, \mathscr{U}_{s}\right)$ is continuous,

ii) $f$ is $\delta$-continuous if and only if $f:\left(X, \mathscr{T}_{s}\right) \rightarrow\left(Y, \mathscr{U}_{s}\right)$ is continuous,

iii) $f$ is $S C$ if and only if $f:\left(X, \mathscr{T}_{s}\right) \rightarrow(Y, \mathscr{U})$ is continuous.

We can prove directly, by using Theorem 4 and Proposition 12, three analogues of the standard result that compactness is a continuous invariant.

Proposition 13. Let $f:(X, \mathscr{T}) \rightarrow(Y, \mathscr{U})$ be a surjection.

i) If $f$ is $A C$ and $(X, \mathscr{T})$ is compact, then $(Y, \mathscr{U})$ is nearly compact.

ii) If $f$ is $\delta$-continuous and $(X, \mathscr{T})$ is nearly compact, then $(Y, \mathscr{U})$ is nearly compact.

iii) If $f$ is $S C$ and $(X, \mathscr{T})$ is nearly compact, then $(Y, \mathscr{U})$ is compact.

These results have appeared as [24, Theorem 3.2], [18, Theorem 5.8] and [13, Theorem 4.1] respectively.

Corresponding to Proposition 12 we make the following definitions. 
DEFINITION 6. A function $f:(X, \mathscr{T}) \rightarrow(Y, \mathscr{U})$ is called

i) almost-open if $f:\left(X, \mathscr{T}_{s}\right) \rightarrow(Y, \mathscr{U})$ is open,

ii) $\delta$-open if $f:\left(X, \mathscr{T}_{s}\right) \rightarrow\left(Y, \mathscr{U}_{s}\right)$ is open,

iii) super-open if $f:(X, \mathscr{T}) \rightarrow\left(Y, \mathscr{U}_{s}\right)$ is open.

Definition 7. A function $f:(X, \mathscr{T}) \rightarrow(Y, \mathscr{U})$ is called

i) star-closed if $f:\left(X, \mathscr{T}_{s}\right) \rightarrow(Y, \mathscr{U})$ is closed,

ii) $\delta$-closed if $f:\left(X, \mathscr{T}_{s}\right) \rightarrow\left(Y, \mathscr{U}_{s}\right)$ is closed,

iii) super-closed if $f:(X, \mathscr{T}) \rightarrow\left(Y, \mathscr{U}_{s}\right)$ is closed.

We note that Singal and Singal [26] defined the classes of almost-open (and almost-closed) maps in terms of images of regular open (regular-closed) sets being open (closed), and Noiri [18] has defined $\delta$-closed maps similarly. Star-closed maps were introduced by Herrington [6], and should not be confused with almost-closed mappings. Each star-closed map is almost-closed but the converse is false.

We can now consider the question of the preservation of the almost locally connected property under appropriate classes of maps, raised by Mancuso [12, Remark 3.22]. By using Theorem 3, Proposition 12 and Definitions 6 and 7, we can obtain three analogues of each of the standard results for local connectedness, namely the continuous open (continuous closed) image of a locally connected space is locally connected.

PROPOSITION 14. Let $f:(X, \mathscr{T}) \rightarrow(Y, \mathscr{U})$ be a surjection.

i) If $f$ is almost-open (or star-closed) and $S C$ and $(X, \mathscr{T})$ is almost locally connected, then $(Y, \mathscr{U})$ is locally connected.

ii) If $f$ is $\delta$-open (or $\delta$-closed) and $\delta$-continuous and $(X, \mathscr{T})$ is almost locally connected, then $(Y, \mathscr{U})$ is almost locally connected.

iii) If $f$ is super-open (or super-closed) and $A C$ and $(X, \mathscr{T})$ is locally connected, then $(Y, \mathscr{U})$ is almost locally connected.

Proposition 15. If $f:(X, \mathscr{T}) \rightarrow(Y, \mathscr{U})$ is $S C$, star-closed and surjective, and $f^{-1}(y)$ is $N$-closed for each point $y \in Y$, then

i) if $(X, \mathscr{T})$ is Hausdorff, $(Y, \mathscr{U})$ is Hausdorff, and

ii) if $(X, \mathscr{T})$ is almost regular, $(Y, \mathscr{U})$ is regular.

Proof. Under the given conditions, $f:\left(X, \mathscr{T}_{s}\right) \rightarrow(Y, \mathscr{U})$ is a perfect map. Now i) follows from Proposition 1 and Dugundji [5, 5.2(1), page 235], while ii) follows from Theorem 1 and [5, 5.2(2), page 235].

Similar proofs yield our final two results. 
Proposition 16. If $f:(X, \mathscr{T}) \rightarrow(Y, \mathscr{U})$ is $\delta$-continuous, $\delta$-closed and surjective, and $f^{-1}(y)$ is $N$-closed for each point $y \in Y$, then

i) if $(X, \mathscr{T})$ is Hausdorff, $(Y, \mathscr{U})$ is Hausdorff, and

ii) if $(X, \mathscr{T})$ is almost regular, $(Y, \mathscr{U})$ is almost regular.

Proposition 17. If $f:(X, \mathscr{T}) \rightarrow(Y, \mathscr{U})$ is $A C$, super-closed and surjective, and if $f^{-1}(y)$ is compact for each point $y \in Y$, then

i) if $(X, \mathscr{T})$ is Hausdorff, $(Y, \mathscr{U})$ is Hausdorff, and

ii) if $(X, \mathscr{T})$ is regular, $(Y, \mathscr{U})$ is almost regular.

\section{References}

[1] N. Bourbaki, Elements of mathematics. General topology. Part I. (Herman, Paris; Addison-Wesley, Reading, Mass., 1966).

[2] D. E. Cameron, 'Properties of $S$-closed spaces', Proc. Amer. Math. Soc. 72 (1978), 581-586.

[3] D. Carnahan, 'Locally nearly compact spaces', Boll. Un. Mat. Ital. (4) 6 (1972), 146-153.

[4] S. G. Crossley, 'A note on semi topological properties', Proc. Amer. Math. Soc. 72 (1978), 409-412.

[5] J. Dugundji, Topology (Allyn and Bacon, Boston, Mass., 1966).

[6] L. L. Herrington, 'Properties of nearly compact spaces', Proc. Amer. Math. Soc. 45 (1974), 431-436.

[7] L. L. Herrington, 'Characterizations of Urysohn-closed spaces', Proc. Amer. Math. Soc. 55 (1976), 435-439.

[8] R. A. Herrman, 'Nearly compact Hausdorff extensions', Glasnik Mat. 12 (32), (1977), 125-132.

[9] R. A. Herrmann, ' $\theta$-rigidity and the idempotent $\theta$-closure', Math. Sem. Notes Kobe Univ. 6 (1978), 217-219.

[10] J. E. Joseph, 'Characterizations of nearly compact spaces', Boll. Un. Mat. Ital. B (5) 13 (1976), 311-321.

[11] M. Katětov, 'Über $H$-abgeschlossene und bikompakte Räume', Časopis Pèst. Mat. 69 (1940), 36-49.

[12] V. J. Mancuso, 'Almost locally connected spaces', J. Austral. Math. Soc. Ser. A 31 (1981), 421-428.

[13] B. M. Munshi and D. S. Bassan, 'Super-continuous mappings', Indian J. Pure Appl. Math. 13 (1982), 229-236.

[14] T. Nieminen, 'On ultra pseudo compact and related spaces', Ann. Acad. Sci. Fenn. Ser. A I Math. 3 (1977), 185-205.

[15] T. Noiri, 'Remarks on locally nearly compact spaces', Boll. Un. Mat. Ital. (4) 10 (1974), 36-43.

[16] T. Noiri, 'A note on locally nearly compact spaces', Bull. Un. Mat. Ital. 12 (1975), 374-380.

[17] T. Noiri, 'Completely continuous images of nearly paracompact spaces', Mat. Vesnik 1(14)(29) (1977), 59-64.

[18] T. Noiri, 'On $\delta$-continuous functions', J. Korean Math. Soc. 16 (1980), 161-166.

[19] P. Papić, 'Sur les espaces presque réguliers', Glasnik Mar. 4(24) (1969), 303-307.

[20] J. Porter and J. Thomas, 'On $H$-closed and minimal Hausdorff spaces', Trans. Amer. Math. Soc. 138 (1969), 159-170.

[21] M. K. Singal and S. P. Arya, 'On nearly paracompact spaces', Mat. Vesnik 6(21) (1969), 3-16.

[22] M. K. Singal and S. P. Arya, 'On almost regular spaces', Glasnik Mat. 4(24) (1969), 89-99.

[23] M. K. Singal and S. P. Arya, 'Almost normal and almost completely regular spaces', Glasnik Mat. 5(25) (1970), 141-152. 
[24] M. K. Singal and A. Mathur, 'On nearly compact spaces', Boll. Un. Mat. Ital. (4) 2 (1969), $702-710$.

[25] M. K. Singal and A. Mathur, 'A note on almost completely regular spaces', Glasnik Mat. 6(26) (1971), 345-349.

[26] M. K. Singal and A. R. Singal, 'Almost continuous mappings', Yokohama Math. J. 16 (1968), 63-73.

[27] L. A. Steen and J. A. Seebach, Counterexamples in topology (Holt, Rinehart and Winston, New York, 1970).

[28] T. Thompson, 'Characterizations of nearly compact spaces', Kyungpook Math. J. 17 (1977), 37-41.

[29] N. V. Velicko, 'H-closed topological space', Mat. Sb. 70(112) (1966), 98-121 (Math. USSR-Sb. (2) 78 (1969), 103-118).

M. Mršević

Institute of Mathematics

Faculty of Sciences

University of Belgrade

Yugoslavia
I. L. Reilly, M. K. Vamanamurthy Department of Mathematics \& Statistics University of Auckland New Zealand 